\title{
The effects of unburned-gas temperature on the characteristics of cellular premixed flames generated by hydrodynamic and diffusive-thermal instabilities in large space: fractal dimension of cellular-flame fronts
}

\author{
Satoshi KADOWAKI*, Taisuke WASHIO**, Thwe Thwe Aung**, Wataru YAMAZAKI**, \\ Toshiyuki KATSUMI** and Hideaki KOBAYASHI*** \\ *Department of System Safety, Nagaoka University of Technology \\ 1603-1 Kamitomioka, Nagaoka 940-2188, Japan \\ E-mail: kadowaki@mech.nagaokaut.ac.jp \\ **Department of Mechanical Engineering, Nagaoka University of Technology \\ 1603-1 Kamitomioka, Nagaoka 940-2188, Japan \\ ***Institute of Fluid Science, Tohoku University \\ 2-1-1 Katahira, Aoba-ku, Sendai 980-8577, Japan
}

Received: 13 April 2017; Revised: 30 May 2017; Accepted: 11 June 2017

\begin{abstract}
The effects of unburned-gas temperature on the characteristics of cellular premixed flames generated by hydrodynamic and diffusive-thermal instabilities were numerically investigated. Two-dimensional reactive flow was calculated in large space, based on the compressible Navier-Stokes equations including a one-step irreversible chemical reaction. The dynamic behavior of cellular premixed flames, i.e. the coalescence and division of cells, appeared in large space owing to intrinsic instability. The behavior of flame fronts became more unstable with a decrease in unburned-gas temperature, even though the burning velocity of a planar flame reduced. This was due to the strength of thermal-expansion effects and to the enlargement of Zeldovich numbers. We found that the average burning velocity of a cellular flame normalized by that of a planar flame increased as the unburned-gas temperature became lower and the space size became larger. To elucidate the increase of burning velocity, we proposed the new model and showed that the normalized increment factor of burning velocity became larger under low unburned-gas temperature. In addition, we performed fractal analysis to consider the fractal dimension for three-dimensional flow. The obtained fractal dimension corresponding to laminar flames was nearly identical to the experimental and numerical results of turbulent flames.
\end{abstract}

Key words : Cellular premixed flame, Dynamic behavior, Intrinsic instability, Unburned-gas temperature, Fractal dimension

\section{Introduction}

To reduce the exergy loss through combustion for energy saving, the excess enthalpy combustion and hightemperature air combustion (or mild combustion) are recognized. The concept of the excess enthalpy combustion was proposed by Weinberg (1971) and by Lloyd and Weinberg (1974), where combustible mixtures were preheated by the heat transfer from hot burned gases. In a series of studies, the concept was successfully extended to the flammable range by the heat recirculation (Takeno et al., 1981; Yoshizawa et al., 1988; Hanamura et al., 1993). In addition, we have knowledge on the high-temperature air combustion, where the mass recirculation, together with heat recirculation, was used (Katsuki and Hasegawa, 1998; Cavaliere and de Joannon, 2004; Miyagoshi et al., 2007; Som and Datta, 2008). Furthermore, lean premixed combustion of hydrogen and/or methane with air receives attention to secure energy source in cold districts. This is because that hydrogen and methane keep gaseous state under low-temperature environments. 
In view of energy saving and energy securing, we need to investigate the characteristics of premixed flames under high- and low-temperature environments, e.g. the intrinsic instability and burning velocity. Planar flames become to cellular flames owing to intrinsic instability, and the burning velocity increases because of the increase in flame-surface area, which are recognized as significant phenomena in premixed flames (Williams, 1985). Hydrodynamic instability, caused by the thermal expansion of combustible mixtures through flame fronts, plays an important role in the intrinsic instability of all premixed flames; diffusive-thermal instability, caused by the preferential diffusion of heat and mass, affects the instability of lean hydrogen-air and methane-air flames. Hydrodynamic and diffusive-thermal instabilities were investigated through experimental, theoretical and numerical approaches, and the mechanism on cellular-flame formation was clarified (Clavin, 1985; Hertzberg, 1989; Sivashinsky, 1990; Kadowaki and Hasegawa, 2005).

Diffusive-thermal instability is closely related with the Lewis-number effects. When the Lewis number is lower (higher) than unity, intrinsic instability becomes stronger (weaker). Thus, the Lewis number has a great influence on the dynamics of premixed flames not only in laminar flow but also in turbulence (Im and Chen, 2002; Chakraborty et al., 2008, 2009), where the direct numerical simulation (DNS) of three-dimensional reactive flow was performed.

In our previous works, we calculated the unsteady reactive flow based on the compressible Navier-Stokes equations to clarify the influence of intrinsic instability on the formation of cellular flames under the low- and high-temperature conditions (Kadowaki et al., 2015). However, large space was not treated but small space in numerical calculations. The numerical results on intrinsic instability will be important information for the industrial application of premixed flames, e.g. premixed-type gas turbines, where combustion phenomena are strongly affected by intrinsic instability (Kobayashi et al., 2007; Ichikawa et al., 2011). Thus, we have to treat reactive flow in large space to obtain the useful knowledge on industrial applications.

The dynamic behavior of cellular fronts in large space were numerically investigated, and the contribution of longwavelength components of disturbances was estimated (Kadowaki et al., 2005, 2016; Frouzakis et al., 2015). However, the characteristics of cellular flames in large space, i.e. the increment factor of burning velocity and the fractal dimension of flame fronts, were not clarified. Obtaining the dependence of burning velocity on unburned-gas temperature and space size, we can propose the new model on the increase of burning velocity. Obtaining the fractal dimension of flame fronts, we can compare with the experimental and numerical results of turbulent premixed flames. Thus, the obtained knowledge is important to the energy saving around the world and the energy securing in cold districts.

In this paper, we performed numerical calculations of two-dimensional reactive flow in large space, based on the compressible Navier-Stokes equations including a one-step irreversible chemical reaction. The effects of unburned-gas temperature on the characteristics of cellular premixed flames were investigated, and the increase of burning velocity was elucidated to propose the new model. Moreover, we obtained the fractal dimension of flame fronts to compare with the previous results.

\section{Basic equations}

We adopted single-reactant flames, where the abundant reactant was excessive and the chemical reaction was controlled only by the deficient reactant, and used the following assumptions: The chemical reaction was a one-step irreversible exothermic reaction, and the reaction rate obeyed the Arrhenius' law. The unburned and burned gases had the same molecular weights and the same Lewis numbers, and the ideal gas equation of state was satisfied. The specific heat and transport coefficients were independent of temperature. The body force, Soret effect, Dufour effect, pressure gradient diffusion, bulk viscosity and heat loss were all negligible.

We employed the compressible Navier-Stokes equations to take account of the thermal-expansion effects which contributed essentially to the intrinsic instability of all premixed flames. The viscous term in the equation of energy conservation was disregarded, because its contribution was trivial in the present problem. Using Cartesian coordinates, we took the direction tangential to the flame front as the $y$-direction, with the gas velocity in the positive $x$-direction. The flow variables were non-dimensionalized by the physical quantities of standard premixed flames, i.e. the density, pressure and temperature of the unburned gas, the burning velocity and the preheat zone thickness.

The basic equations of two-dimensional reactive flow were written in the conservation form as 


$$
\begin{aligned}
& \frac{\partial}{\partial t}\left(\begin{array}{c}
\rho \\
\rho u \\
\rho v \\
\rho Y
\end{array}\right)+\frac{\partial}{\partial x}\left(\begin{array}{c}
\rho u \\
\rho u^{2}+\frac{p}{\gamma M_{0}^{2}}-\operatorname{Pr}\left(\frac{4}{3} \frac{\partial u}{\partial x}-\frac{2}{3} \frac{\partial v}{\partial y}\right) \\
\rho u v-\operatorname{Pr}\left(\frac{\partial v}{\partial x}+\frac{\partial u}{\partial y}\right) \\
(e+p) u-\frac{\gamma}{\gamma-1} \frac{\partial T}{\partial x} \\
\rho Y u-\frac{1}{L e} \frac{\partial Y}{\partial x}
\end{array}\right) \\
& +\frac{\partial}{\partial y}\left(\begin{array}{c}
\rho v \\
\rho u v-\operatorname{Pr}\left(\frac{\partial v}{\partial x}+\frac{\partial u}{\partial y}\right) \\
(e+p) v-\frac{\gamma}{\gamma-1} \frac{\partial T}{\partial y} \\
\rho Y v-\frac{1}{L e} \frac{\partial Y}{\partial y} \\
\left.0 . \frac{4}{3} \frac{\partial v}{\partial y}-\frac{2}{3} \frac{\partial u}{\partial x}\right) \\
0 \\
0 \\
\gamma \quad Q B \rho Y \exp \left(-\frac{E}{T}\right) \\
-B \rho Y \exp \left(-\frac{E}{T}\right)
\end{array}\right)
\end{aligned}
$$

where $\rho$ is the density, $u$ and $v$ are the velocities in the $x$ - and $y$-directions, $e$ is the stored energy, $Y$ is the mass fraction of fuel, $p$ is the pressure, $T$ is the temperature, $\gamma$ is the ratio of two specific heats, $M_{0}$ is the Mach number, Pr is the Prandtl number, $L e$ is the Lewis number, $Q$ is the heating value, $B$ is the frequency factor and $E$ is the activation energy. In addition, the ideal gas equation of state, $p=\rho T$, is satisfied.

\section{Calculation procedures}

We simulated a standard premixed flame whose burning velocity and adiabatic flame temperature were $3.93 \mathrm{~m} / \mathrm{s}$ and $2086 \mathrm{~K}$, respectively, at room temperature and atmospheric pressure. Although the adopted burning velocity was considerably large, this value was sufficiently small compared with the sound velocity. Thus, we obtained the same results on intrinsic instability even though the burning velocity changed. Adopting a large burning velocity, we completed numerical calculations in a short time.

The used parameters were $\gamma=1.4, M_{0}=0.01, \operatorname{Pr}=1.0, Q=6.0$ and $E=70$. The non-dimensional activation energy corresponded to the activation energy of $173 \mathrm{~kJ} / \mathrm{mol}$. The frequency factor was determined under the conditions that the calculated burning velocity of a planar flame should be equal to unity at the unburned-gas temperature $T_{\mathrm{u}}=1.0$. We took $L e=0.5$ to take account of hydrodynamic and diffusive-thermal instabilities. To investigate the effects of unburned-gas temperature, we took $T_{\mathrm{u}}=0.6$ through 1.5 under constant pressure, where the burned-gas temperature was 6.6 through 7.5 .

The basic equations of two-dimensional reactive flow were solved by the explicit MacCormack scheme. The boundary conditions were provided by free-flow conditions in the $x$-direction and by periodic conditions in the $y$ direction. The computational domain in the $x$-direction $L_{x}$ was 400 times the preheat zone thickness. In the $y$ direction, we set $L_{\mathrm{y}}$ to one to sixteen times the wavelength of a disturbance $\lambda$. A uniformly spaced grid was used. The grid size in the $x$-direction was 0.2 , and the grid size in the $y$-direction was $\lambda / 64$. The computational domain was resolved by a $2001 \times 65$ grid for dispersion relations and by a $2001 \times 1025$ grid for cellular flames. The used grid was 
fine enough to perform numerical calculations on this type of problem. When the size of grid was halved, no significant difference was observed. A time-step interval was adopted to satisfy the Courant-Friedrichs-Lewy (CFL) condition, and the non-dimensional calculation time was $t=200$.

We superimposed a disturbance periodic in the $y$-direction on a planar flame and calculated the evolution of the disturbed flame front. The displacement of flame front in the $x$-direction due to the superimposed disturbance was

$$
a_{\mathrm{i}} \sin (2 \pi y / \lambda)
$$

The initial amplitude $a_{\mathrm{i}}$ was set to 0.1 for dispersion relations and to 1.0 for cellular flames.

\section{Dispersion relations}

To investigate the intrinsic instability of premixed flames, a sinusoidal disturbance with sufficiently small amplitude ( $a_{\mathrm{i}}=0.1$ ) was superimposed on a planar flame. The amplitude of a disturbance grew exponentially with time, $a \sim \exp$ $(\omega t)$, where $\omega$ is called the growth rate. The behavior of the disturbed flame front, i.e. growing exponentially with time, was consistent with the prediction of previous linear analyses, and was observed only for sufficiently small amplitude. When the superimposed disturbance grew to some degree, the growth rate was gradually lowered. This was due to the nonlinearity brought about by finite amplitude.

The growth rate of a disturbance depends on the wavelength, i.e. on the wave number $k$, and the relation between the growth rate and wave number is called the dispersion relation. Figure 1 shows the dispersion relations at $L e=0.5$, depending on the unburned-gas temperature, where hydrodynamic and diffusive-thermal instabilities appear. As the unburned-gas temperature becomes lower, the growth rate decreases and the unstable range narrows. This is due mainly to the reduction of the burning velocity of a planar flame. The dispersion relation yields the wave number corresponding to the maximum growth rate, i.e. the linearly most unstable wave number, and we refer to as the critical wave number $k_{\mathrm{c}}$. The lower the unburned-gas temperature, the smaller the critical wave number. Thus, the critical wavelength $\lambda_{\mathrm{c}}(=2 \pi$ $/ k_{\mathrm{c}}$ ) expands as the unburned-gas temperature becomes lower.

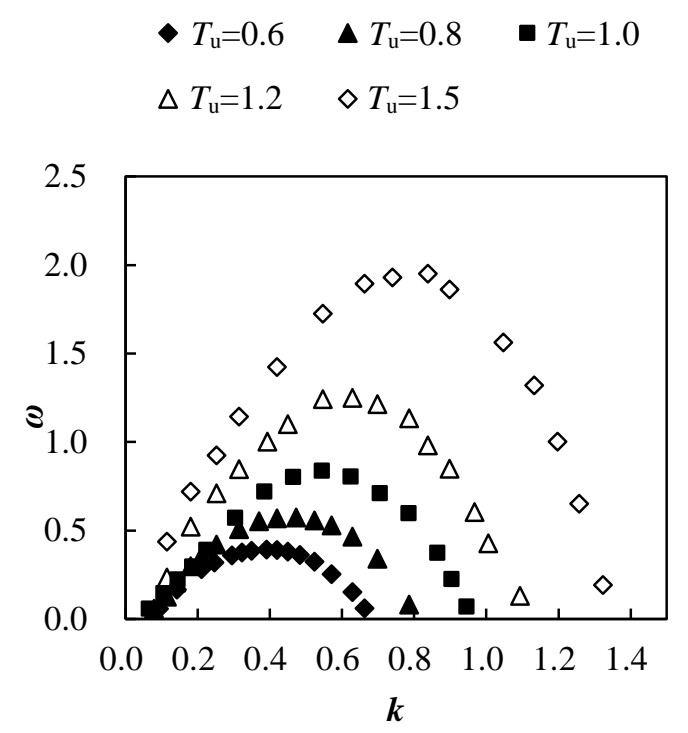

Fig. 1. Dispersion relations at $L e=0.5$, depending on the unburned-gas temperature.

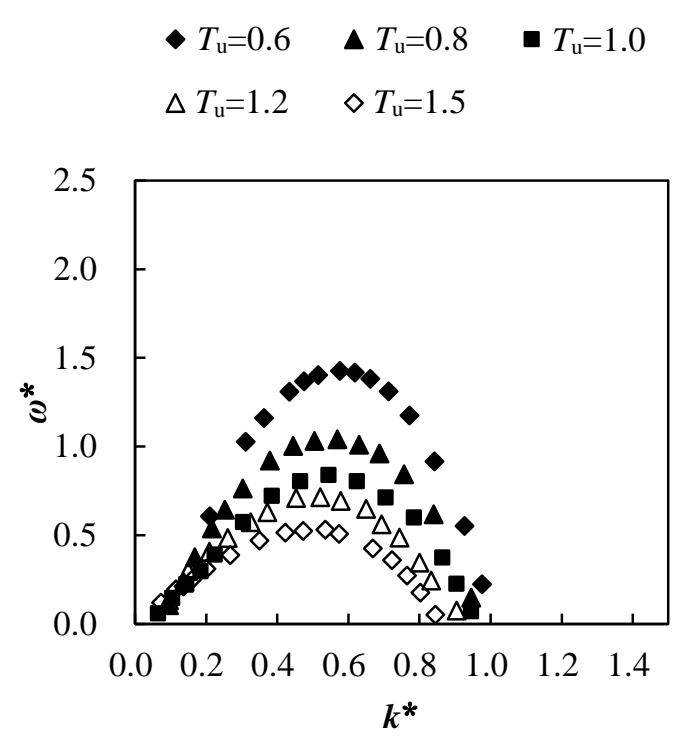

Fig. 2. Normalized dispersion relations at $L e=0.5$, depending on the unburned-gas temperature. 
The growth rate and wave number depend on the preheat zone thickness which is inversely proportional to the mass flux density $m_{\mathrm{u}}$, and on the burning velocity of a planar flame $S_{\mathrm{u}}$. Thus, the growth rate is proportional to $m_{\mathrm{u}} S_{\mathrm{u}}$; the wave number is proportional to $m_{\mathrm{u}}$ (Pelce and Clavin, 1982; Kadowaki et al., 2015). To investigate the effects of unburned-gas temperature on instability phenomena essentially, we normalize the growth rate and wave number. The normalized dispersion relations, i.e. the relation between the normalized growth rate $\omega^{*}\left(=\omega / m_{\mathrm{u}} S_{\mathrm{u}}\right)$ and normalized wave number $k^{*}\left(=k / m_{\mathrm{u}}\right)$, are shown in Fig. 2. As the unburned-gas temperature becomes lower, the normalized growth rate increases and the normalized unstable range widens. This is because that hydrodynamic instability becomes stronger owing to the increase of the temperature ratio of burned and unburned gases, and that diffusive-thermal instability becomes greater owing to the enlargement of Zeldovich numbers (Sivashinsky, 1977). In the present investigation, the temperature ratios at $T_{\mathrm{u}}=0.6,1.0$ and 1.5 are 11.0, 7.0 and 5.0, respectively, and the Zeldovich numbers (Niioka, 2004) are 9.64, 8.57 and 7.47, respectively.

\section{Cellular flames}

To investigate the characteristics of cellular flames induced by intrinsic instability, we superimposed a finite disturbance $\left(a_{\mathrm{i}}=1.0\right)$ with the critical wavelength on a planar flame. Although smaller disturbances were superimposed, we obtained the same results on the dynamic behavior of cellular flames.

The temperature distributions of cellular flames at $L e=0.5, L_{\mathrm{y}}=16 \lambda_{\mathrm{c}}$ and $T_{\mathrm{u}}=0.8,1.0$ and 1.2 are shown in Fig. 3. At $T_{\mathrm{u}}=0.8,1.0$ and $1.2, \lambda_{\mathrm{c}}=13.30,11.55$ and 10.00, respectively, so that $L_{\mathrm{y}}=212.8,184.8$ and 160.0 , respectively. The unburned gas flows in from the left, and the burned gas flows out to the right. In all cases, the superimposed disturbances evolve, and then cellular flames form, which is due to hydrodynamic and diffusive-thermal instabilities. The cellular front shape is drastically changed with time, and several small cells on large cells are observed. The combination of small cells emerges, and then the evolution and division of large cells also appear. After that, we find again the coalescence and division of cells. In downstream of convex fronts toward to the unburned gas, the burnedgas temperature is higher than the adiabatic flame temperature of a planar flame, which is because of the diffusive-thermal effects (Clavin, 1985). This overshoot of temperature is the significant factor of dynamic behaviors. Moreover, the behavior of flame fronts becomes more unstable with a decrease in unburned-gas temperature, and the depth of cells becomes larger. This is because that the thermal-expansion effects become stronger, and that the change of local burning velocity at convex and concave fronts becomes greater.

Owing to the coalescence and division of cells, the number of cells and cell width change drastically with time. Figure 4 shows the time histories of the number of cells $N_{\text {cell, }}$ and the ratio of cell width to critical wavelength $W_{\text {cell }} / \lambda_{\text {c }}$. The number of cells becomes larger with a decrease in unburned-gas temperature. Average numbers of cells at $T_{\mathrm{u}}=0.8$, 1.0 and 1.2 are 17.7, 16.5 and 12.2, respectively. In addition, the ratio of cell width to critical wavelength decreases. Average ratios at $T_{\mathrm{u}}=0.8,1.0$ and 1.2 are $1.43,1.67$ and 1.76 , respectively. We find small ratios under low unburnedgas temperature, which is due to high instability intensity.

Cellular flames have larger surface area, so that the burning velocity of a cellular flame is larger than that of a planar flame. In the present calculations, the burning velocity of a cellular flame is obtained as follows: The reaction rate is integrated throughout the computational domain, and the integrated value is divided by that of a planar flame at $T_{\mathrm{u}}=1.0$. This value is consistent with the burning velocity of a cellular flame. Figure 5 shows the average burning velocities of cellular flames $S_{\mathrm{cf}}$ normalized by those of planar flames at $L e=0.5$ and $T_{\mathrm{u}}=0.6,0.8,1.0,1.2$ and 1.5 , depending on $L_{\mathrm{y}} / \lambda_{\mathrm{c}}$. In this figure, we show the relation between $S_{\mathrm{cf}} / S_{\mathrm{u}}$ and $L_{\mathrm{y}} / \lambda_{\mathrm{c}}$ to investigate the effects of space size on cellular instability essentially, because the critical wavelength, i.e. the typical length of intrinsic instability, depends on the unburned-gas temperature. Adopting the normalized space size $L_{y} / \lambda_{c}$, we can clarify the effects of space size on the increase of burning velocity generated by intrinsic instability. As the unburned-gas temperature becomes lower, the average burning velocity of a cellular flame normalized by that of a planar flame increases. In addition, the burning velocity of a cellular flame increases monotonously as the normalized space size becomes larger. This denotes that the unburnedgas temperature and long-wavelength components of disturbances have a great influence on the dynamic behavior of cellular flames.

We estimate quantitatively the increase of burning velocity of a cellular flame. We propose the new model as follows: 
Kadowaki, Washio, Thwe Thwe Aung, Yamazaki, Katsumi and Kobayashi, Journal of Thermal Science and Technology, Vol.12, No.1 (2017)
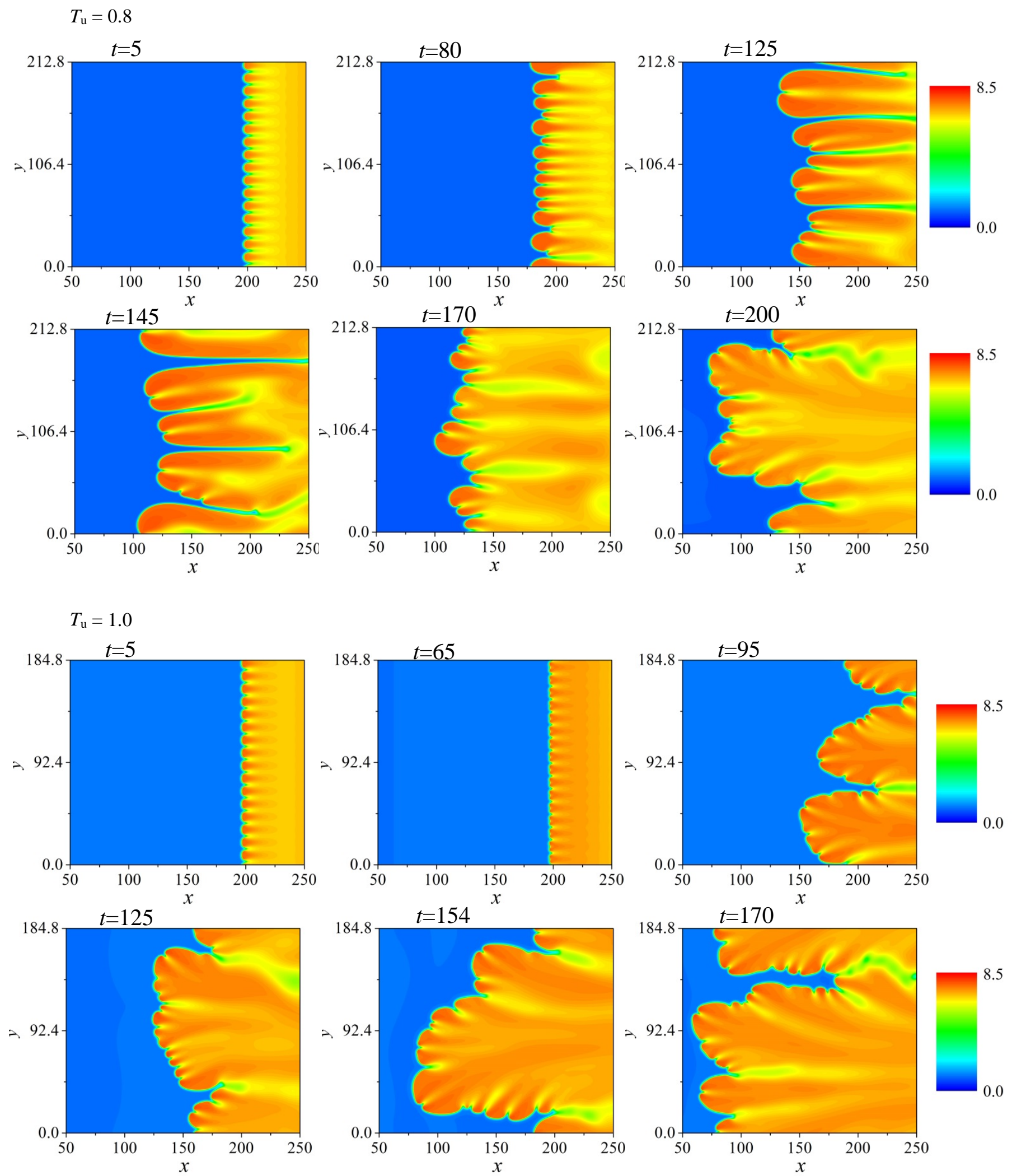

Fig. 3. Temperature distributions of cellular flames at $L e=0.5, L_{\mathrm{y}}=16 \lambda_{\mathrm{c}}$ and $T_{\mathrm{u}}=0.8,1.0$ and 1.2 (to be continued). 
$T_{\mathrm{u}}=1.2$
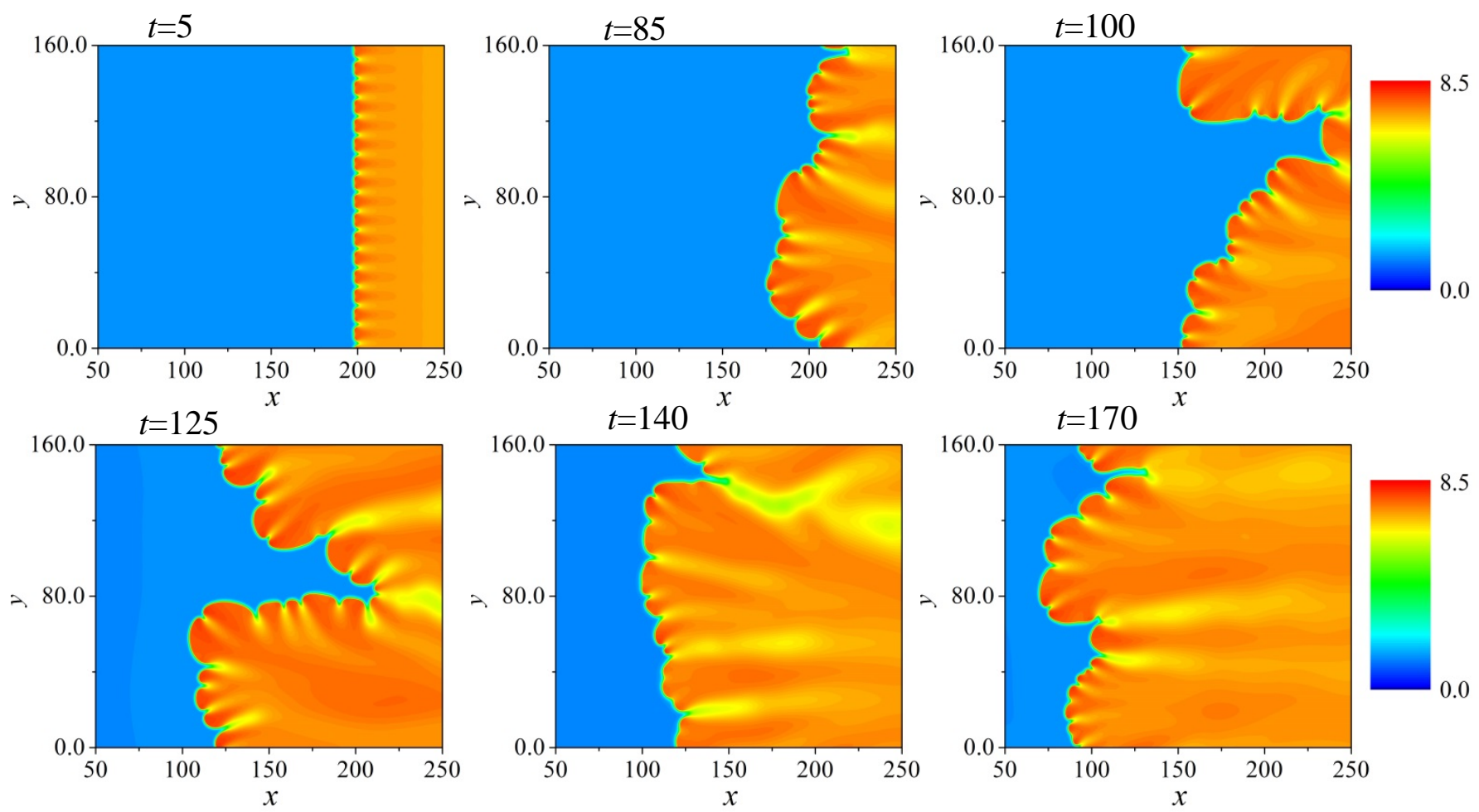

Fig. 3. Temperature distributions of cellular flames at $L e=0.5, L_{\mathrm{y}}=16 \lambda_{\mathrm{c}}$ and $T_{\mathrm{u}}=0.8,1.0$ and 1.2.
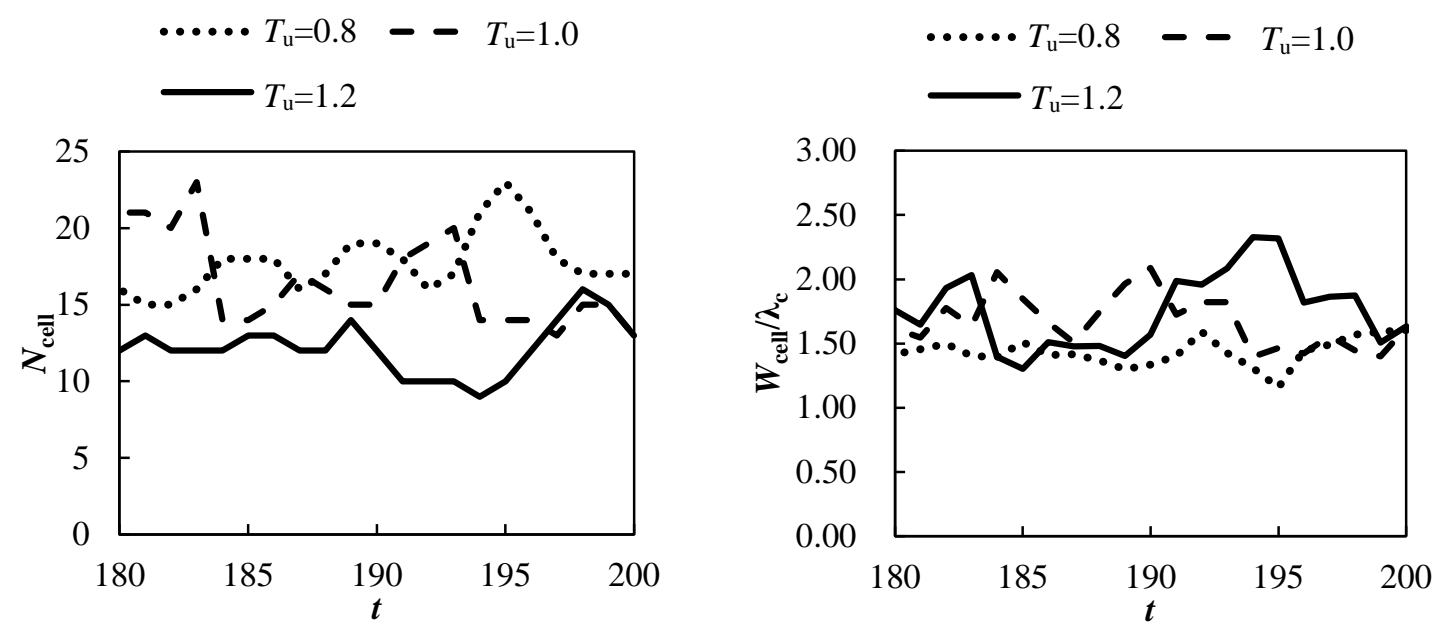

Fig. 4. Time histories of the number of cells (left) and the ratio of cell width to critical wavelength (right) at $L e=0.5$, $L_{\mathrm{y}}=16 \lambda_{\mathrm{c}}$ and $T_{\mathrm{u}}=0.8,1.0$ and $1.2(t=180-200)$. 

- $T_{\mathrm{u}}=0.6 \quad \Delta T_{\mathrm{u}}=0.8 \quad$ - $T_{\mathrm{u}}=1.0$
$\Delta T_{\mathrm{u}}=1.2 \quad \diamond T_{\mathrm{u}}=1.5$

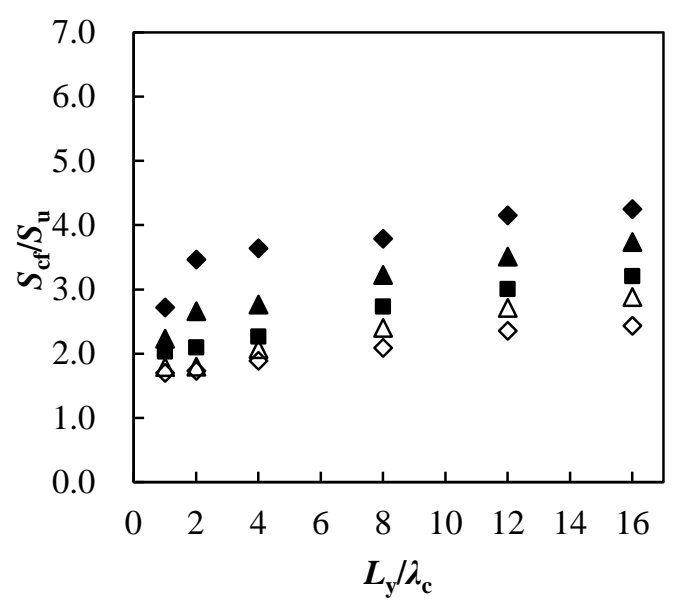

Fig. 5. Average burning velocities of cellular flames normalized by those of planar flames at $L e=0.5$ and $T_{\mathrm{u}}=0.6,0.8,1.0,1.2$ and 1.5 , depending on $L_{\mathrm{y}} / \lambda_{\mathrm{c}}$.

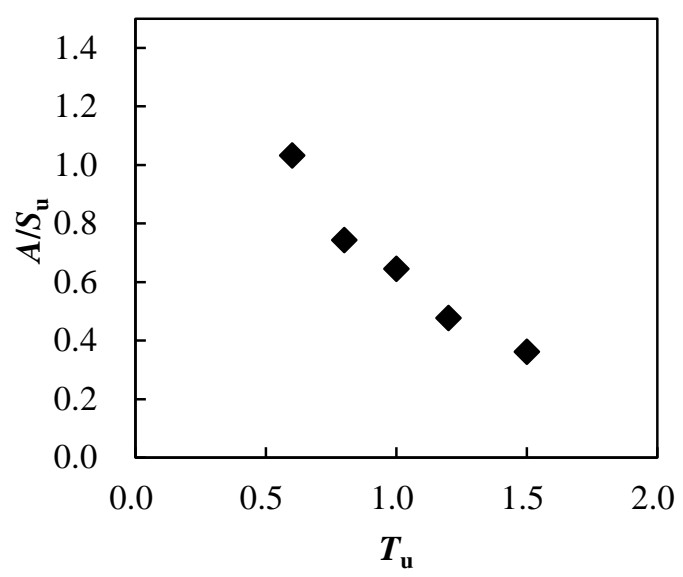

Fig. 6. Increment factors of burning velocity normalized by the burning velocities of planar flames at $L e=0.5$, depending on the unburned-gas temperature.

$$
\frac{S_{\mathrm{cf}}}{S_{\mathrm{u}}}=\frac{A}{S_{\mathrm{u}}} \ln \left(\frac{L_{\mathrm{y}}}{\lambda_{\mathrm{m}}}\right)+1
$$

where $A$ is the increment factor of burning velocity and $\lambda_{\mathrm{m}}$ is the marginal wavelength for neutral stability. This relation is valid for $L_{\mathrm{y}}>\lambda_{\mathrm{m}}$. Figure 6 shows the increment factors normalized by the burning velocities of planar flames at $L e$ $=0.5$, depending on the unburned-gas temperature. The normalized increment factor becomes larger under low unburned-gas temperature. This is due to the strength of thermal-expansion effects and to the enlargement of Zeldovich numbers.

Finally, we perform fractal analysis to obtain the fractal dimension of flame fronts. Using the binary image of reaction rate of cellular flames shown in Fig.7, we obtain the fractal dimension through the box-counting method (Miyauchi et al., 1994; Mukaiyama et al., 2013). The fractal dimension is estimated by the relation between the number of circles $N_{\mathrm{r}}$ and the radius of circles $r$ for cellular flames (see Fig. 8). The fractal dimension for two-dimensional flow $d$ is obtained by the following relation:

$$
d=-\frac{\log \left(N_{r}\right)}{\log (r)}
$$

From the gradients of lines shown in Fig.8, we obtain the fractal dimension which is independent of time, $d=1.20$ at $T_{\mathrm{u}}=1.0$. In addition, it is confirmed that the fractal dimension is independent of the space size when $L_{\mathrm{y}}$ is larger than $8 \lambda_{c}$. The fractal dimension extracted from two-dimensional fronts depends on the unburned-gas temperature. As the unburned-gas temperature becomes lower, the fractal dimension increases (see Fig. 9). The results indicate that the flame front becomes increasingly complex when the unburned-gas temperature is low. We compare with the previous results of numerical calculations based on the model equation, i.e. the Sivashinsky equation. Compared with the results of Mukaiyama et al. (2013), the present fractal dimensions are slightly large except for the $T_{\mathrm{u}}=1.5$ flame. This is because that the present calculations are based on the compressible Navier-Stokes equations.

We consider the fractal dimension for three-dimensional flow $D$, which is estimated as follows:

$$
D=d+1
$$



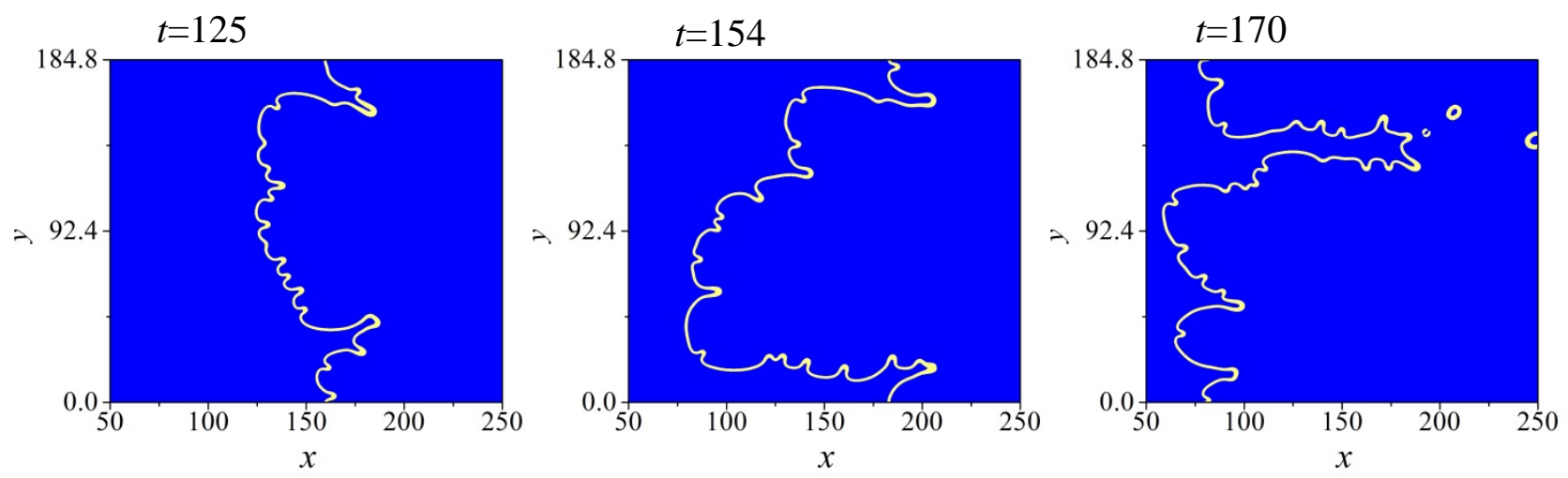

Fig. 7. Binary images of reaction rate of cellular flames at $L e=0.5, L_{\mathrm{y}}=16 \lambda_{\mathrm{c}}$ and $T_{\mathrm{u}}=1.0(t=125,154$ and 170$)$.

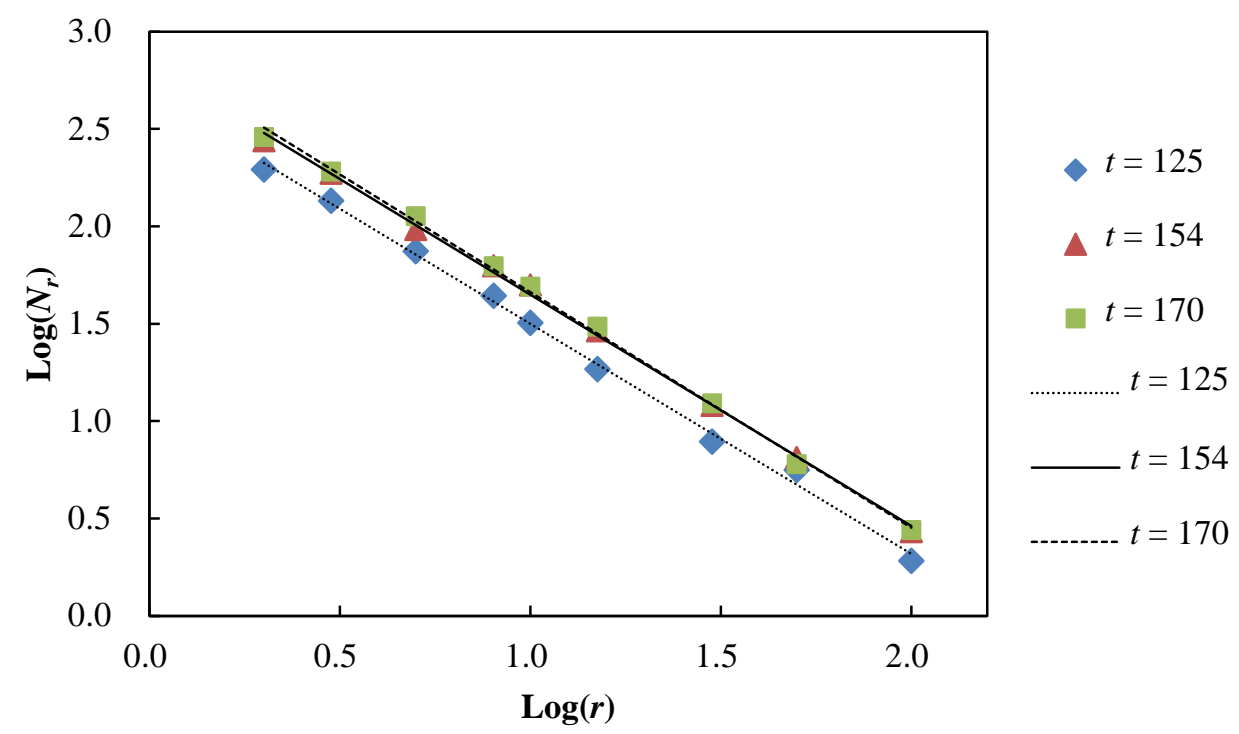

Fig. 8. Relations between $N_{r}$ and $r$ for cellular flames at $L e=0.5, L_{\mathrm{y}}=16 \lambda_{\mathrm{c}}$ and $T_{\mathrm{u}}=1.0(t=125,154$ and 170$)$.

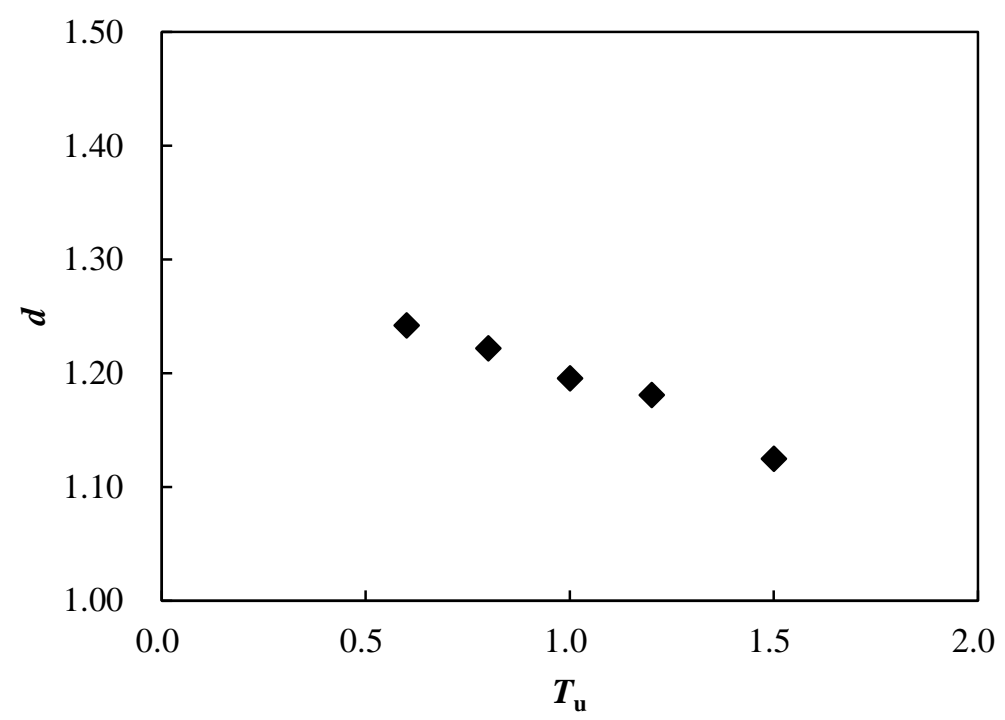

Fig. 9. Fractal dimensions of flame fronts at $L e=0.5$ and $L_{\mathrm{y}}=16 \lambda_{\mathrm{c}}$, depending on the unburned-gas temperature. 
The value obtained by Eq. (5) is almost equal to the fractal dimension directly obtained by three-dimensional boxcounting method (Battista, 2015). Therefore, we estimate $D=2.20$ at $T_{\mathrm{u}}=1.0$ in the present investigation. In the previous investigation, the fractal dimension of turbulent premixed flames was estimated experimentally and numerically, i.e. $D=2.23 \pm 0.03$, and it was reported that the fractal dimension is almost constant despite the level of turbulent intensity and is smaller than that of non-reacting turbulence (Battista, 2015). The present results corresponding to laminar flames are nearly identical to the experimental and numerical results of turbulent flames. These indicate that the fractal dimension would be governed not by turbulent property but by flame characteristics.

\section{Conclusions}

In this paper, we have performed numerical calculations of two-dimensional reactive flow, based on the compressible Navier-Stokes equations, to investigate the effects of unburned-gas temperature on the characteristics of cellular premixed flames in large space. The obtained results are as follows:

As the unburned-gas temperature becomes lower, the growth rate decreases and the unstable range narrows. On the other hand, the normalized growth rate increases and the normalized unstable range widens, which is due to the strength of thermal-expansion effects and to the enlargement of Zeldovich numbers. Moreover, the dynamic behavior of cellular premixed flames, i.e. the coalescence and division of cells, appears in large space owing to hydrodynamic and diffusivethermal instabilities. The behavior of flame fronts becomes more unstable with a decrease in unburned-gas temperature, and the number of cells and depth of cells become larger. In addition, the average burning velocity of a cellular flame normalized by that of a planar flame increases as the unburned-gas temperature becomes lower and the space size becomes larger. We propose the new model on the increase of burning velocity of a cellular flame and show that the normalized increment factor of burning velocity becomes larger under low unburned-gas temperature. Furthermore, we consider the fractal dimension for three-dimensional flow, corresponding to laminar flames. The obtained results are nearly identical to the experimental and numerical results of turbulent flames, indicating that the fractal dimension would be governed not by turbulent property but by flame characteristics.

In the present investigation, we treat the two-dimensional reactive flow. In the near future, we will try threedimensional calculations to compare the flame characteristics between two- and three-dimensional fields. In addition, we hope the experiments under high- and low-temperature environments will be performed to reveal details of the temperature effects on the flame characteristics.

\section{References}

Battista, F., Troiani, G. and Picano, F., Fractal scaling of turbulent premixed flame fronts: application to LES, International Journal of Heat and Fluid Flow, Vol. 51 (2015), pp. 78-87.

Cavaliere, A. and de Joannon, M., Mild combustion, Progress in Energy and Combustion Science, Vol. 30 (2004), pp. 329-366.

Chakraborty, N., Hawkes, E. R., Chen, J. H. and Cant, R. S., The effects of strain rate and curvature on surface density function transport in turbulent premixed methane-air and hydrogen-air flames: a comparative study, Combustion and Flame, Vol. 154 (2008), pp. 259-280.

Chakraborty, N., Klein, M. and Swaminathan, N., Effects of Lewis number on the reactive scalar gradient alignment with local strain rate in turbulent premixed flames, Proceedings of the Combustion Institute, Vol. 32 (2009), pp. 14091417.

Clavin, P., Dynamic behavior of premixed flame fronts in laminar and turbulent flows, Progress in Energy and Combustion Science, Vol. 11 (1985), pp. 1-59.

Frouzakis, C. E., Fogla, N., Tomboulides, A. G., Altantzis, C. and Matalon, M., Numerical study of unstable hydrogen/air flames: shape and propagation speed, Proceedings of the Combustion Institute, Vol. 35 (2015), pp. 1087-1095.

Hanamura, K., Echigo, R. and Zhdanok, S. A., Superadiabatic combustion in a porous medium, International Journal of Heat and Mass Transfer, Vol. 36 (1993), pp. 3201-3209.

Hertzberg, M., Selective diffusional demixing: occurrence and size of cellular flames, Progress in Energy and Combustion Science, Vol. 15 (1989), pp. 203-239. 
Ichikawa, Y., Otawara, H., Kobayashi, H., Ogami, Y., Kudo, T., Okuyama, M. and Kadowaki, S., Flame structure and radiation characteristics of $\mathrm{CO} / \mathrm{H}_{2} / \mathrm{CO}_{2} /$ air turbulent premixed flames at high pressure, Proceedings of the Combustion Institute, Vol. 33 (2011), pp. 1543-1550.

Im, H. G. and Chen, J. H., Preferential diffusion effects on the burning rate of interacting turbulent premixed hydrogenair flames, Combustion and Flame, Vol. 131 (2002), pp. 246-258.

Kadowaki, S. and Hasegawa, T., Numerical simulation of dynamics of premixed flames: flame instability and vortexflame interaction, Progress in Energy and Combustion Science, Vol. 31 (2005), pp. 193-241.

Kadowaki, S., Suzuki, H. and Kobayashi, H., The unstable behavior of cellular premixed flames induced by intrinsic instability, Proceedings of the Combustion Institute, Vol. 30 (2005), pp. 169-176.

Kadowaki, S., Yanagioka, T., Yamazaki, W. and Kobayashi, H., The intrinsic instability of three-dimensional premixed flames under the low- and high-temperature conditions: effects of unburned-gas temperature on hydrodynamic and diffusive-thermal instabilities, Combustion Science and Technology, Vol. 187 (2015), pp. 1167-1181.

Kadowaki, S., Washio, T., Thwe Thwe Aung, Yamazaki, W., Katsumi, T. and Kobayashi, H., Unstable behaviors of cellular premixed flames caused by hydrodynamic and diffusive-thermal instabilities under high- and lowtemperature environment, Transactions of the JSME (in Japanese), Vol.82, No.835, (2016), DOI: 10.1299/ transjsme.15-00522.

Katsuki, M. and Hasegawa, T., The science and technology of combustion in highly preheated air, Proceedings of the Combustion Institute, Vol. 27 (1998), pp. 3135-3146.

Kobayashi, H., Hagiwara, H., Kaneko, H. and Ogami, Y., Effects of $\mathrm{CO}_{2}$ dilution on turbulent premixed flames at high pressure and high temperature, Proceedings of the Combustion Institute, Vol. 31 (2007), pp. 1451-1458.

Lloyd, S. A. and Weinberg, F. J., A burner for mixtures of very low heat content, Nature, Vol. 251 (1974), pp. 47-49.

Miyagoshi, Y., Tatefuku, T., Nishino, M., Yokoyama, T. and Kadowaki, S., Advantages of low air-ratio combustion in a stoker-type incinerator, Journal of Environment and Engineering, Vol. 2 (2007), pp. 183-193.

Miyauchi, T., Tanahashi, M. and Gao, F., Fractal characteristics of turbulent diffusion flames, Combustion Science and Technology, Vol. 96 (1994), pp. 135-154.

Mukaiyama, K., Shibayama, S. and Kuwana, K., Fractal structures of hydrodynamically unstable and diffusive-thermally unstable flames, Combustion and Flame, Vol. 160 (2013), pp. 2471-2475.

Niioka, T., Analysis of Combustion Phenomena (2004), p. 5, Tohoku University Press.

Pelce, P. and Clavin, P., Influence of hydrodynamic and diffusion upon the stability limits of laminar premixed flames, Journal of Fluid Mechanics, Vol. 124 (1982), pp. 219-237.

Som, S. K. and Datta, A., Thermodynamic irreversibilities and exergy balance in combustion processes, Progress in Energy and Combustion Science, Vol. 34 (2008), pp. 351-376.

Sivashinsky, G. I., Diffusional-thermal theory of cellular flames, Combustion Science and Technology, Vol. 15 (1977), pp. 137-146.

Sivashinsky, G. I., On the intrinsic dynamics of premixed flames, Philosophical Transactions of the Royal Society of London, Series A, Vol. 332 (1990), pp. 135-148.

Takeno, T., Sato, K. and Hase, K., A theoretical study on an excess enthalpy flame, Proceedings of the Combustion Institute, Vol. 18 (1981), pp. 465-472.

Yoshizawa, Y., Sasaki, K. and Echigo, R., Analytical study of the structure of radiation controlled flame, International Journal of Heat and Mass Transfer, Vol. 31 (1988), pp. 311-319.

Weinberg, F. J., Combustion temperature: the future?, Nature, Vol. 233 (1971), pp. 239-241.

Williams, F. A., Combustion Theory, Second Edition (1985), p. 349, Addison-Wesley Publishing Company. 